\title{
A Note on the Singularities of Residue Currents of Integrally Closed Ideals
}

\section{Elizabeth Wulcan ${ }^{1}$}

Received: 5 January 2019 / Revised: 19 February 2019 / Accepted: 22 February 2019 /

Published online: 20 August 2019

(C) The Author(s) 2019

\section{Abstract}

Given a free resolution of an ideal $\mathfrak{a}$ of holomorphic functions, there is an associated residue current $R$ that coincides with the classical Coleff-Herrera product if $\mathfrak{a}$ is a complete intersection ideal and whose annihilator ideal equals $\mathfrak{a}$. In the case when $\mathfrak{a}$ is an Artinian monomial ideal, we show that the singularities of $R$ are small in a certain sense if and only if $\mathfrak{a}$ is integrally closed.

Keywords Residue currents · Integrally closed ideals $\cdot$ Monomial ideals

Mathematics Subject Classification (2010) 13B22 - 13D02 - 32A27 · 32S45

\section{Introduction}

Given (a germ of) a holomorphic function $f$ at $0 \in \mathbf{C}^{n}$, Herrera and Lieberman [11] proved that one can define the principal value current

$$
\frac{1}{f} \cdot \xi:=\lim _{\epsilon \rightarrow 0} \int_{|f|^{2}>\epsilon} \frac{\xi}{f},
$$

for test forms $\xi$. It follows that $\bar{\partial}(1 / f)$ is a current with support on the variety of $f$; such a current is called a residue current. The duality principle asserts that a holomorphic germ $g$ is in the ideal generated by $f$ if and only if $g \bar{\partial}(1 / f)=0$.

Given a (locally) free resolution

$$
0 \rightarrow E_{N} \stackrel{\varphi_{N}}{\longrightarrow} E_{N-1} \rightarrow \cdots \rightarrow E_{1} \stackrel{\varphi_{1}}{\longrightarrow} E_{0} \rightarrow 0
$$

of a general ideal (sheaf) $\mathfrak{a}$, in [3] with Andersson, we defined a vector (bundle) valued residue current $R$ with support on the variety of $\mathfrak{a}$ that satisfies the duality principle for $\mathfrak{a}$, cf. Section 2.2 below. If $\mathfrak{a}$ is Cohen-Macaulay, then $R$ is essentially independent of the

Elizabeth Wulcan

wulcan@chalmers.se

1 Department of Mathematical Sciences, Chalmers University of Technology and University of Gothenburg, SE-412 96 Gothenburg, Sweden 
resolution. In particular, if $(E, \varphi)$ is the Koszul complex of a minimal set of generators $f_{1}, \ldots, f_{p}$ of a complete intersection ideal, then $R$ coincides with the classical ColeffHerrera product [9],

$$
\bar{\partial} \frac{1}{f_{p}} \wedge \cdots \wedge \bar{\partial} \frac{1}{f_{1}} .
$$

By means of these residue currents, we were able to extend several results previously known for complete intersections. These currents have also turned out to be particularly useful for analysis on singular spaces; for example, they have been used to obtain new results on the $\bar{\partial}$-equation [2] and new global versions of the classical Briançon-Skoda theorem [5] on singular spaces.

In view of the duality principle, the residue current $R$ can be thought of as a current representing the ideal $\mathfrak{a}$; this idea is central to many applications of residue currents, including the ones mentioned above. Various properties of the ideal $\mathfrak{a}$ are reflected in the residue current $R$. For example, $R$ has a natural geometric decomposition corresponding to a primary decomposition of $\mathfrak{a}$, see [4], and the fundamental cycle of $\mathfrak{a}$ admits a natural representation in terms of $R$ that generalizes the classical Poincaré-Lelong formula, see [14].

In this note we study the singularities of $R$ and show that, for a monomial ideal $\mathfrak{a}$, they are small in a certain sense if and only if $\mathfrak{a}$ is integrally closed. For simplicity, we will work in a local setting; let $\mathcal{O}_{0}^{n}$ be the ring of germs of holomorphic functions at $0 \in \mathbf{C}^{n}$ and let $\mathfrak{a}$ be an ideal in $\mathcal{O}_{0}^{n}$. Recall that $g \in \mathcal{O}_{0}^{n}$ is in the integral closure $\overline{\mathfrak{a}}$ of $\mathfrak{a}$ if $|g| \leq C|f|$, where $C$ is a constant and $f$ is a set of generators $f_{1}, \ldots, f_{m} \in \mathcal{O}_{0}^{n}$ of $\mathfrak{a}$, or equivalently if $g$ satisfies a monic equation $g^{q}+h_{1} g^{q-1}+\cdots+h_{q}=0$, where $h_{k} \in \mathfrak{a}^{k}$. If $\overline{\mathfrak{a}}=\mathfrak{a}$, then $\mathfrak{a}$ is said to be integrally closed. Assume that $\pi: \widetilde{X} \rightarrow\left(\mathbf{C}^{n}, 0\right)$ is a log-resolution of a, i.e., $\widetilde{X}$ is a complex manifold, $\pi$ is a biholomorphism outside the variety of $\mathfrak{a}$, and $\mathfrak{a} \cdot \mathcal{O}_{\tilde{X}}=\mathcal{O}_{\widetilde{X}}(-D)$, where $D=\sum_{i=1}^{N} r_{i} D_{i}$ is an effective divisor with simple normal crossings support. Then $\overline{\mathfrak{a}}=\pi_{*}\left(\mathcal{O}_{\widetilde{X}}(-D)\right)$, which means that $g \in \mathcal{O}_{0}^{n}$ is in $\overline{\mathfrak{a}}$ if and only if $\operatorname{ord}_{D_{i}}(g) \geq r_{i}$ for each $i$, where $\operatorname{ord}_{D_{i}}$ denotes the divisorial valuation defined by the prime divisor $D_{i}$.

If $\pi: \widetilde{X} \rightarrow\left(\mathbf{C}^{n}, 0\right)$ is a common log-resolution of $\mathfrak{a}$ and the Fitting ideals of $\mathfrak{a}$, i.e., the ideals generated by the minors of optimal rank of the $\varphi_{k}$ in (1.2), then there is a section $\sigma$ of a line bundle $L=\mathcal{O}_{\widetilde{X}}(-F)$ over $\widetilde{X}$ and a current $\widetilde{R}$ on $\widetilde{X}$ such that

$$
\widetilde{R} \wedge \pi^{*} d z=\eta \wedge \bar{\partial} \frac{1}{\sigma},
$$

where $d z=d z_{1} \wedge \cdots \wedge d z_{n}$ and $\eta$ is a vector (bundle) valued smooth form with values in $L$, such that $\pi_{*} \widetilde{R}=R$, see [3, Section 2] and Section 2.2 below. The observation that residue currents in this way can be seen as pushforwards of residue currents of principal ideal sheaves is crucial for many applications of residue currents, cf. Section 2.1 below.

Assume that

$$
\sigma=\sigma_{1}^{a_{1}} \cdots \sigma_{N}^{a_{N}}
$$

where $\sigma_{i}$ are holomorphic sections of line bundles $\mathcal{O}\left(-D_{i}\right)$ defining the prime divisors $D_{i}$ of $F=\sum_{i=1}^{N} a_{i} D_{i}$. We are interested in the exponents $a_{i}$. Naively, one could hope that one could choose $a_{i}$ as $r_{i}=\operatorname{ord}_{D_{i}}(\mathfrak{a})$. However, this can only be true if $\mathfrak{a}$ is integrally closed. Indeed, assume that $R=\pi_{*} \widetilde{R}$, where $\widetilde{R}$ satisfies (1.4) with $\sigma$ given by (1.5) with $a_{i} \leq r_{i}$. Take $g \in \overline{\mathfrak{a}}$; then $\operatorname{ord}_{D_{i}}(g) \geq r_{i}$ for each $i$ and thus $\pi^{*} g=\sigma g^{\prime}$, where $g^{\prime}$ is a holomorphic section of $L^{-1}$. Therefore, by the duality principle, $\pi^{*} g \bar{\partial}(1 / \sigma)=0$ and so $\pi^{*} g \widetilde{R}=0$, which implies that $g R=0$, and hence $g \in \mathfrak{a}$. To conclude, if we can choose $a_{i} \leq r_{i}$ for each $i$, then $\mathfrak{a}$ is integrally closed.

We are interested in whether the converse holds, i.e., if $\mathfrak{a}$ is integrally closed, is it then always possible to find an $\widetilde{R}$ as above with $\sigma$ given by (1.5) with $a_{i} \leq r_{i}$ ? In this note, we 
answer this question affirmatively when $R$ is the residue current associated with a cellular resolution, introduced by Bayer-Sturmfels [8], see Section 2.3 below, of an Artinian, i.e., 0 -dimensional, monomial ideal, and when we moreover allow $\eta$ to be semi-meromorphic, i.e., of the form $(1 / f) \omega$, where $\omega$ is smooth and $f$ is holomorphic. For the definition of the product $\eta \wedge \mu$, where $\eta$ is a semi-meromorphic form and $\mu$ is a residue current, or more generally a so-called pseudomeromorphic current, see Section 2.1 below. Multiplication from the left by $\eta$ does not increase the singularities in the sense that if $g$ is a holomorphic function such that $g \mu=0$, then $g \eta \wedge \mu=0$.

Theorem 1.1 Let $M \subset \mathcal{O}_{0}^{n}$ be an integrally closed Artinian monomial ideal and let $R$ be the residue current associated with a cellular resolution of $M$. Then there is a log-resolution $\pi: \widetilde{X} \underset{\sim}{\longrightarrow}\left(\mathbf{C}_{\tilde{X}}^{n}, 0\right)$ of $M$, such that $M \cdot \mathcal{O}_{\widetilde{X}}=\mathcal{O}_{\widetilde{X}}(-D)$, where $D=\sum_{i=1}^{N} r_{i} D_{i}$, and a current $\widetilde{R}$ on $\widetilde{X}$ such that $\pi_{*} \widetilde{R}=R$ and

$$
\widetilde{R} \wedge \pi^{*} d z=\eta \wedge \bar{\partial} \frac{1}{\sigma_{1}^{r_{1}} \cdots \sigma_{N}^{r_{N}}},
$$

where $\sigma_{i}$ is a holomorphic section defining $D_{i}$ and $\eta$ is a semi-meromorphic form.

The proof uses explicit descriptions of residue currents of monomial ideals, [19], as well as so-called Bochner-Martinelli residue currents, [13], cf. Sections 2.3 and 2.6 below, and it should be possible to extend to general, not necessarily Artinian, monomial ideals. There is a brief discussion of this and other aspects of our result at the end of Section 3.

\section{Preliminaries}

\subsection{Pseudomeromorphic Currents}

To get a coherent approach to principal value and residue currents, in [4] with Andersson, we introduced the sheaf of pseudomeromorphic currents which essentially are pushforwards of tensor products of principal value and residue currents times smooth forms, like

$$
\frac{1}{s_{2}^{b_{2}} \cdots s_{m}^{b_{m}}} \omega \wedge \bar{\partial} \frac{1}{s_{1}^{b_{1}}},
$$

where $s_{1}, \ldots, s_{m}$ are (local) coordinates in some $\mathbf{C}^{m}$ and $\omega$ is a smooth form. Principal value currents and the residue currents mentioned in this paper are typical examples of pseudomeromorphic currents.

Pseudomeromorphic currents have a geometric nature similar to positive closed currents. For example, the dimension principle states that if the pseudomeromorphic current $\mu$ has bidegree $(*, p)$ and support on a variety of codimension larger than $p$, then $\mu$ vanishes. Moreover if $\mu$ is a pseudomeromorphic current and $\mathbf{1}_{V}$ is the characteristic function of an analytic variety $V$, then the product $\mathbf{1}_{V} \mu$, defined through a suitable regularization, is a well-defined pseudomeromorphic current with support on $V$, see [4, Proposition 2.2].

A current of the form $(1 / f) \omega$ where $f$ is a holomorphic section of a line bundle $L \rightarrow X$ and $\omega$ is a smooth form with values in $L$ is said to be semi-meromorphic. If $\eta$ is a semi-meromorphic form, or more generally the pushforward under a modification of a semi-meromorphic form, and $\mu$ is a pseudomeromorphic current, there is a unique pseudomeromorphic current $\eta \wedge \mu$ that coincides with the usual product where $\eta$ is smooth and such that $\mathbf{1}_{Z S S(\eta)} \eta \wedge \mu=0$, where $Z S S(\eta)$ is the smallest analytic set containing the set 
where $\eta$ is not smooth, see, e.g., [6, Section 4.2]. If $h$ is a holomorphic tuple such that $\{h=0\}=Z S S(\eta)$ and $\chi(t)$ is (a smooth approximand of) the characteristic function of the interval $[1, \infty)$, then

It follows that for $c>0$

$$
\eta \wedge \mu=\lim _{\epsilon \rightarrow 0} \chi\left(|h|^{2} / \epsilon\right) \eta \wedge \mu .
$$

$$
\frac{1}{s_{i}^{c}} \frac{1}{s_{i}^{b}}=\frac{1}{s_{i}^{b+c}}, \quad \frac{1}{s_{i}^{c}} \bar{\partial} \frac{1}{s_{i}^{b}}=0 .
$$

For further reference, in view of (1.1), note that

$$
s_{i}^{c} \frac{1}{s_{i}^{b}}=\frac{1}{s_{i}^{b-c}}, \quad s_{i}^{c} \bar{\partial} \frac{1}{s_{i}^{b}}=\bar{\partial} \frac{1}{s_{i}^{b-c}} .
$$

Example 2.1 Assume that $s_{1}, \ldots, s_{n}$ are (local) coordinates in $\mathbf{C}^{n}$. If $D=\left\{s_{1}=0\right\}$, by the dimension principle,

$$
\mathbf{1}_{D} \bar{\partial} \frac{1}{s_{1}^{b_{1}} \cdots s_{n}^{b_{n}}}=\frac{1}{s_{2}^{b_{2}} \cdots s_{n}^{b_{n}}} \bar{\partial} \frac{1}{s_{1}^{b_{1}}} .
$$

In view of (2.1) and (2.2), it follows that for $c_{1} \geq b_{1}$ and any choices of $c_{2}, \ldots, c_{n}$,

$$
\mathbf{1}_{D} \bar{\partial} \frac{1}{s_{1}^{b_{1}} \cdots s_{n}^{b_{n}}}=\mathbf{1}_{D} s_{1}^{c_{1}-b_{1}} \cdots s_{n}^{c_{n}-b_{n}} \bar{\partial} \frac{1}{s_{1}^{c_{1}} \cdots s_{n}^{c_{n}}},
$$

where the factor $s_{i}^{c_{i}-b_{i}}$ should be understood as a principal value if $c_{i}<b_{i}$.

\subsection{Residue Currents from Complexes of Vector Bundles}

Let

$$
0 \rightarrow E_{N} \stackrel{\varphi_{N}}{\longrightarrow} E_{N-1} \rightarrow \cdots \rightarrow E_{1} \stackrel{\varphi_{1}}{\longrightarrow} E_{0} \rightarrow 0
$$

be a complex of Hermitian vector bundles over a complex manifold $X$ of dimension $n$ that is exact outside a variety $Z \subset X$. In [3] with Andersson, we constructed an $\operatorname{End}\left(\oplus E_{k}\right)$ valued residue current $R$ with support on $Z$ that in some sense measures the exactness of the associated sheaf complex

$$
0 \rightarrow \mathcal{O}\left(E_{N}\right) \stackrel{\varphi_{N}}{\longrightarrow} \mathcal{O}\left(E_{N-1}\right) \rightarrow \cdots \rightarrow \mathcal{O}\left(E_{1}\right) \stackrel{\varphi_{1}}{\longrightarrow} \mathcal{O}\left(E_{0}\right) \rightarrow 0
$$

of holomorphic sections. If (2.4) is exact, then $R$ satisfies the duality principle, which means that if $\xi$ is a section of $E_{0}$ that is generically in the image of $\varphi_{1}$, then $R \xi=0$ if and only if $\xi \in \operatorname{Im} \varphi_{1}$; in particular, if (2.4) is a free resolution of an ideal $\mathfrak{a} \subset \mathcal{O}_{0}^{n}$, then the annihilator ideal of $R$, i.e., the ideal of germs of holomorphic functions $g$ such that $g R=0$, equals $\mathfrak{a}$. Moreover, then $R$ is of the form $R=\sum R_{k}$, where $R_{k}$ is a $\operatorname{Hom}\left(E_{0}, E_{k}\right)$-valued pseudomeromorphic current of bidegree $(0, k)$. Note that $R_{k}$ vanishes for $k<\operatorname{codim} Z$ by the dimension principle, and for $k>n$ for degree reasons. In particular, if (2.4) is a free resolution of an Artinian monomial ideal in $\mathcal{O}_{0}^{n}$, then $R=R_{n}$.

Let $\rho_{k}$ be the optimal rank of $\varphi_{k}$, and let $\pi: \widetilde{X} \rightarrow X$ be a common log-resolution of the ideal sheaves generated by the $\rho_{k}$-minors of the $\varphi_{k}$, i.e., such that the pullback of the section $\operatorname{det}^{\rho_{k}} \varphi_{k}$ of $\Lambda^{\rho_{k}} E_{k}^{*} \otimes \Lambda^{\rho_{k}} E_{k-1}$ is of the form $t_{k} \rho_{k}^{\prime}$, where $t_{k}$ is a section of some line bundle $L_{k}$ and $\varphi_{k}^{\prime}$ is a nonvanishing section of $L_{k}^{-1} \otimes \Lambda^{\rho_{k}} \pi^{*} E_{k}^{*} \otimes \Lambda^{\rho_{k}} \pi^{*} E_{k-1}$. It was proved in [3, Section 2] that there is a current $\widetilde{R}$ on $\widetilde{X}$ such that $\pi_{*} \widetilde{R}=R$ and $\widetilde{R}=\omega \wedge \bar{\partial}(1 / \sigma)$, where $\omega$ is smooth and $\sigma=t_{1} \cdots t_{\min (n, N)}$. The form $\omega$ may vanish along the divisor $F$ of $\sigma$, and thus in general it may be possible to find a $\sigma$ that vanishes to lower order along $F$ than $t_{1} \cdots t_{\min (n, N)}$, cf. Example 2.3 below. 


\subsection{Monomial Ideals and Cellular Resolutions}

Let us briefly recall the construction of cellular resolutions due to Bayer-Sturmfels, [8]. Let $M$ be a monomial ideal in the polynomial ring $S:=\mathbf{C}\left[z_{1}, \ldots, z_{n}\right]$, i.e., $M$ is generated by monomials $m_{1}, \ldots, m_{r}$ in $S$. Moreover, let $K$ be an oriented polyhedral cell complex, where the vertices are labeled by the generators $m_{i}$ and the face $\tau$ of $K$ is labeled by the least common multiple $m_{\tau}$ of the labels $m_{i}$ of the vertices of $\tau$. Then with $K$ there is an associated graded complex of $S$-modules. For $k=0, \ldots, \operatorname{dim} K+1$, let $A_{k}$ be the free $S$ module with one generator $e_{\tau}$ in degree $m_{\tau}$ for each $\tau \in K_{k}$, where $K_{k}$ denotes the faces of $K$ of dimension $k-1\left(K_{0}\right.$ should be interpreted as $\left.\{\varnothing\}\right)$ and let $\varphi_{k}: A_{k} \rightarrow A_{k-1}$ be defined by

$$
\varphi_{k}: e_{\tau} \mapsto \sum_{\tau^{\prime} \subset \tau} \operatorname{sgn}\left(\tau^{\prime}, \tau\right) \frac{m_{\tau}}{m_{\tau^{\prime}}} e_{\tau^{\prime}},
$$

where $\operatorname{sgn}\left(\tau^{\prime}, \tau\right)$ is a sign coming from the orientation of $K$. Now the complex $(A, \varphi)$ is exact precisely if the labeled polyhedral cell complex $K$ satisfies a certain acyclicity condition, see [8, Proposition 1.2]. We then say that the complex $(A, \varphi)$ is a cellular resolution of $M$. Any monomial ideal admits a cellular resolution, cf. [8, Proposition 1.5].

Let $M$ denote also the monomial ideal of germs of holomorphic functions at $0 \in \mathbf{C}_{z_{1}, \ldots, z_{n}}^{n}$ generated by the monomials $m_{1}, \ldots, m_{r}$. Since $\mathcal{O}_{0}^{n}$ is flat over $S,(A, \varphi)$ induces a free resolution of $M \subset \mathcal{O}_{0}^{n}$. More precisely, for $k=0, \ldots, N=\operatorname{dim} K+1$, let $E_{k}$ be a trivial bundle over (a neighborhood of 0 in) $\mathbf{C}^{n}$ with a global frame $\left\{e_{\tau}\right\}_{\tau \in K_{k}}$, endowed with the trivial metric, and where the differential $\varphi_{k}$ is given by (2.5). Then (2.4) is exact if $(A, \varphi)$ is. We will think of monomial ideals sometimes as ideals in $S$, sometimes as ideals in $\mathcal{O}_{0}^{n}$, and sometimes as ideals in the ring of entire functions in $\mathbf{C}^{n}$.

In [19], we computed the residue current $R$ of a cellular resolution of a monomial ideal $M$. Note that if $M$ is Artinian, then $R=R_{n}$ is of the form $R=\sum R_{\tau} e_{\tau} \otimes e_{\emptyset \emptyset}^{*}$, i.e., with one component for each $\tau \in K_{n}$. Proposition 3.1 in that paper asserts that if $z^{\alpha}:=z_{1}^{\alpha_{1}} \cdots z_{n}^{\alpha_{n}}$ is the label of $\tau$, then $R_{\tau}=c_{\tau} R_{\alpha}$, where $c_{\tau} \in \mathbf{C}$ and

$$
R_{\alpha}=\bar{\partial} \frac{1}{z_{n}^{\alpha_{n}}} \wedge \cdots \wedge \bar{\partial} \frac{1}{z_{1}^{\alpha_{1}}} .
$$

\subsection{Toric Log-Resolutions}

For an (Artinian) monomial ideal $M$ in $\mathbf{C}^{n}$, it is possible to find a log-resolution $\pi: \widetilde{X} \rightarrow$ $\mathbf{C}^{n}$ where $\tilde{X}$ is a toric variety. Let us briefly recall this construction, which can be found, e.g., in [7, p. 82]. For a general reference on toric varieties, see, e.g., [10]. A (rational strongly convex) cone in $\mathbf{R}^{n}$ is a set of the form $\mathcal{C}=\sum \mathbf{R}_{+} v_{i}$, where $v_{i}$ are in the lattice $\mathbf{Z}^{n}$, that contains no line; here $\mathbf{R}_{+}$denotes the non-negative real numbers. A cone is regular if the $v_{i}$ can be chosen as part of a basis for the lattice $\mathbf{Z}^{n}$. A fan $\Delta$ is a finite collection of cones such that all faces and intersections of cones in $\Delta$ are in $\Delta ; \Delta$ is regular if all cones are regular. A regular fan $\Delta$ determines a smooth toric variety $X(\Delta)$, obtained by patching together affine toric varieties corresponding to the cones in $\Delta$.

Assume that $M$ is an Artinian monomial ideal in $\mathbf{C}_{z_{1}, \ldots, z_{n}}^{n}$. Recall that the Newton polyhedron $\mathrm{NP}(M)$ of $M$ is defined as the convex hull in $\mathbf{R}^{n}$ of the exponents of monomials in $M$. Let $\mathcal{S}(M)$ be the collection of cones of the form $\mathcal{C}=\mathbf{R}_{+} \rho \subset \mathbf{R}_{+}^{n}$, where $\rho$ is a normal vector of a compact facet (face of maximal dimension) of $\mathrm{NP}(M)$. Let $\Delta$ be a regular fan that contains $\mathcal{S}(M)$ and such that the support, i.e., the union of all cones in $\Delta$, equals $\mathbf{R}_{+}^{n}$. The cones in $\mathcal{S}(M)$ determine a fan with support $\mathbf{R}_{+}^{n}$ and by refining this is always possible 
to find such a $\Delta$. Then $\pi: X(\Delta) \rightarrow \mathbf{C}^{n}$ is a log-resolution of $M$. The prime divisors $D_{i}$ of the exceptional divisor correspond to one-dimensional cones $\mathcal{C}_{i}=\mathbf{R}_{+} \rho^{i}$ in $\Delta$ and $\operatorname{ord}_{D_{i}}$ are monomial valuations (i.e., determined by its values on $z_{1}, \ldots, z_{n}$ ). More precisely, if $\rho=\left(\rho_{1}, \ldots, \rho_{n}\right)$ is the first non-zero lattice point met along $\mathcal{C}_{i}$, then $\operatorname{ord}_{D_{i}}$ is the monomial valuation $\operatorname{ord}_{\rho}\left(z_{1}^{a_{1}} \cdots z_{n}^{a_{n}}\right):=\rho_{1} a_{1}+\cdots+\rho_{n} a_{n}$.

\subsection{Rees Valuations}

Given a non-zero ideal $\mathfrak{a} \subset \mathcal{O}_{0}^{n}$, let $v: X^{+} \rightarrow\left(\mathbf{C}^{n}, 0\right)$ be the normalized blow-up of $\mathfrak{a}$ and let $D=\sum r_{i} D_{i}$ be the exceptional divisor, such that $\mathfrak{a} \cdot \mathcal{O}_{X^{+}}=\mathcal{O}_{X^{+}}(-D)$. The divisorial valuations ord $D_{D_{i}}$ are called the Rees valuations of $\mathfrak{a}$, see, e.g., [15, Section 9.6.A]. Then $\overline{\mathfrak{a}}=\nu_{*}\left(\mathcal{O}_{X^{+}}(-D)\right)$, i.e., $g \in \overline{\mathfrak{a}}$ if and only if $\operatorname{ord}_{D_{i}}(g) \geq \operatorname{ord}_{D_{i}}(\mathfrak{a})$ for each $i$. If $\pi: \widetilde{X} \rightarrow\left(\mathbf{C}^{n}, 0\right)$ is any log-resolution of $\mathfrak{a}$ (and thus factors through the normalized blowup) with exceptional divisor $D=\sum r_{i} D_{i}$, following [13] we say that the prime divisor $D_{i}$ is a Rees divisor if $\operatorname{ord}_{D_{i}}$ is a Rees valuation.

Let $M$ be an Artinian monomial ideal (at 0) in $\mathbf{C}_{z_{1}, \ldots, z_{n}}^{n}$. Then the Rees valuations are monomial and in one-to-one correspondence with the compact facets of $\operatorname{NP}(M)$. We say that the normal vector $\rho$ of a facet $\tau$ is primitive if it has minimal non-negative entries, i.e., if $\rho$ is the first lattice point met along the cone $\mathbf{R}_{+} \rho \subset \mathbf{R}_{+}^{n}$. If $\rho$ is a primitive normal vector of a compact facet $\tau$, then the Rees valuation corresponding to $\tau$ is the monomial valuation $\operatorname{ord}_{\rho}$, see, e.g., [12, Theorem 10.3.5] and [7, p. 82]. It follows that in the toric log-resolution $\pi: X(\Delta) \rightarrow \mathbf{C}^{n}$ in the previous section, $D_{i}$ is a Rees divisor of $M$ if and only if the corresponding cone $\mathcal{C}_{i}$ is in $\mathcal{S}(M)$.

Example 2.2 Given $\beta=\left(\beta_{1}, \ldots, \beta_{n}\right) \in \mathbf{N}^{n}$, we let $\mathfrak{m}^{\beta}$ denote the Artinian monomial complete intersection ideal generated by $z_{1}^{\beta_{1}}, \ldots, z_{n}^{\beta_{n}}$. Then $\mathrm{NP}\left(\mathfrak{m}^{\beta}\right)$ has a unique compact facet, namely the simplex $\tau$ with vertices $\left(\beta_{1}, 0 \ldots, 0\right),\left(0, \beta_{2}, 0, \ldots, 0\right), \ldots,\left(0, \ldots, 0, \beta_{n}\right)$. Let $\rho_{j}=\beta_{1} \cdots \beta_{j-1} \beta_{j+1} \cdots \beta_{n}$; then $\rho=\left(\rho_{1}, \ldots, \rho_{n}\right)$ is a normal vector of $\tau$ and thus the unique Rees valuation of $\mathfrak{m}^{\beta}$ is of the form $r \operatorname{ord}_{\rho}$ for some $r \in \mathbf{Q}$. Note that $\operatorname{ord}_{\rho}\left(z_{i}^{\beta_{i}}\right)=$ $\operatorname{ord}_{\rho}\left(\mathfrak{m}^{\beta}\right)$ for all $i$.

\subsection{Bochner-Martinelli Residue Currents}

Let $f=\left(f_{1}, \ldots, f_{p}\right)$ be a tuple (of germs) of holomorphic functions at $0 \in \mathbf{C}^{n}$ and let (2.3) be the Koszul complex of $f$, i.e., consider $f$ as a section $f=\sum f_{j} e_{j}^{*}$ of a trivial rank $p$ bundle $E^{*}$ over (a neighborhood of 0 in) $\mathbf{C}^{n}$ with a frame $e_{1}^{*}, \ldots, e_{p}^{*}$, let $E_{j}=\bigwedge^{j} E$, where $E$ is the dual bundle of $E^{*}$, and let $\varphi_{k}=\delta_{f}$ be contraction with $f$. Assume that the complex is equipped with the trivial metric. Then the coefficients of the associated residue current are the so-called Bochner-Martinelli residue currents introduced by Passare et al. [17]. In particular, if $f_{1}, \ldots, f_{p}$ are minimal generators of a complete intersection ideal, then the only nonvanishing coefficient of $R=R_{p}$ equals the Coleff-Herrera product (1.3), see [17, Theorem 4.1] and [1, Theorem 1.7].

In [13], together with Jonsson, we gave a geometric description of the residue current $R$ in this case in terms of the Rees valuations of the ideal $\mathfrak{a}=\mathfrak{a}(f)$ generated by $f$. It is proved in Section 4 in that paper that if $\pi: \widetilde{X} \rightarrow\left(\mathbf{C}^{n}, 0\right)$ is a log-resolution of $\mathfrak{a}$, then there is a current $\widetilde{R}$ such that $\pi_{*} \widetilde{R}=R$ and $\widetilde{R}$ has support on the Rees divisors of $\mathfrak{a}$. Moreover if 
$D=\sum_{i=1}^{N} r_{i} D_{i}$ is the exceptional divisor of $\pi$, then

$$
\widetilde{R}=\omega \wedge \bar{\partial} \frac{1}{\sigma_{1}^{n r_{1}} \cdots \sigma_{N}^{n r_{N}}},
$$

where $\sigma_{i}$ is a holomorphic section defining $D_{i}$ and $\omega$ is a smooth form.

Example 2.3 Let $\mathfrak{a}_{\ell}=\left(z_{1}^{\ell}, \ldots, z_{n}^{\ell}\right) \subset \mathcal{O}_{0}^{n}$, and let (2.3) be the Koszul complex of $\left(z_{1}^{\ell}, \ldots, z_{n}^{\ell}\right)$. Then the $\rho_{k}$-minors of the $\varphi_{k}$ are monomials of degree $\rho_{k} \ell$. It follows that the blow-up of $\mathbf{C}^{n}$ at 0 is a common $\log$-resolution of $\mathfrak{a}_{\ell}$ and the ideals generated by the $\rho_{k}$-minors of the $\varphi_{k}$. Let $D=\left\{\sigma_{1}=0\right\}$ denote the exceptional (prime) divisor. Then $\operatorname{ord}_{D}\left(z_{i}\right)=1$ for each $i$ and $\operatorname{ord}_{D}(d z)=n-1$. It follows that the section $t_{k}$ from Section 2.2 is of the form $t_{k}=\sigma_{1}^{\rho_{k} \ell}$, so that according to Section 2.2 there is an $\widetilde{R}$ that satisfies (1.4) with $\sigma=\sigma_{1}^{\left(\rho_{1}+\cdots+\rho_{n}\right) \ell-n+1}$ and where $\eta$ is smooth. However, noting that $\operatorname{ord}_{D}\left(\mathfrak{a}_{\ell}\right)=\ell$, in view of (2.7), we can, in fact, choose $\widetilde{R}$ with $\sigma=\sigma_{1}^{(n-1) \ell+1}$.

\section{Proof of Theorem 1.1}

Theorem 1.1 is a direct consequence of the following slightly more precisely formulated result.

Theorem 3.1 Let $M \subset \mathcal{O}_{0}^{n}$ be an integrally closed Artinian monomial ideal and let $R$ be the residue current associated with a cellular resolution of $M$ corresponding to the labeled polyhedral cell complex $K$. Then there is a log-resolution $\pi: \widetilde{X} \underset{\sim}{\sim}\left(\mathbf{C}^{n}, 0\right)$ of $M$ and a current $\widetilde{R}$ on $\widetilde{X}$ with support on the Rees divisors of $M$ such that $\pi_{*} \widetilde{R}=R$ and $\widetilde{R} \wedge \pi^{*} d z$ is of the form (1.6), where $D=\sum r_{\tilde{\sim}} D_{i}, \sigma_{i}$, and $\eta$ are as in Theorem 1.1. More precisely, for each $\tau \in K_{\tilde{\sim}}$, there is a current $\widetilde{R}_{\tau}$ on $\widetilde{X}$ and a Rees divisor $D_{\tau}$ such that $\widetilde{R}_{\tau}$ has support on $D_{\tau}, \pi_{*} \widetilde{R}_{\tau}=R_{\tau}$, and

$$
\widetilde{R}_{\tau} \wedge \pi^{*} d z=\eta_{\tau} \wedge \bar{\partial} \frac{1}{\sigma_{1}^{r_{1}} \cdots \sigma_{N}^{r_{N}}},
$$

where $\eta_{\tau}$ is a semi-meromorphic form.

Proof Let $\pi: \widetilde{X} \rightarrow\left(\mathbf{C}^{n}, 0\right)$ be a toric log-resolution of $M$ in the sense of Section 2.4. Consider an entry $R_{\tau}=c_{\tau} R_{\alpha}$ of $R$, where $c_{\tau} \neq 0$, cf. Section 2.3. Note that $z^{\alpha-1} R_{\alpha} \neq 0$, where $\mathbf{1}=(1, \ldots, 1)$. It follows that $z^{\alpha-1} R_{\tau} \neq 0$, and thus $z^{\alpha-1} R \neq 0$, which by the duality principle implies that $z^{\alpha-1} \notin M$. Since $M$ is integrally closed, there is a Rees divisor $D_{\tau}$, that we may assume equals $D_{1}$, of $M=\bar{M}$ such that

$$
\operatorname{ord}_{D_{1}}\left(z^{\alpha-1}\right)<\operatorname{ord}_{D_{1}}(M),
$$

see Section 2.5.

Since $M$ is monomial, $\operatorname{ord}_{D_{1}}$ is a monomial valuation of the form $\operatorname{ord}_{\rho}$, where $\rho=$ $\left(\rho_{1}, \ldots, \rho_{n}\right)$ is the primitive normal vector of one of the compact facets of $\mathrm{NP}(M)$; in particular, $\rho_{j} \in \mathbf{N}$, see Sections 2.4 and 2.5. Let $\gamma_{j}=\rho_{1} \cdots \rho_{j-1} \rho_{j+1} \cdots \rho_{n}$ and choose $k \in \mathbf{N}$ such that $\beta_{j}:=k \gamma_{j} \geq \alpha_{j}$ for all $j$. Then $\rho$ is the primitive normal vector of the unique compact facet of the Newton polyhedron of $\mathfrak{m}^{\beta}=\left(z_{1}^{\beta_{1}}, \ldots, z_{n}^{\beta_{n}}\right)$, so that $D_{1}$ is the unique Rees divisor of $\mathfrak{m}^{\beta}$, see Example 2.2. It follows that $\pi: \tilde{X} \rightarrow\left(\mathbf{C}^{n}, 0\right)$ is a $\log$-resolution of $\mathfrak{m}^{\beta}$, see Section 2.4. Recall from Section 2.6 that (the coefficient of) the 
Bochner-Martinelli residue current of $\left(z_{1}^{\beta_{1}}, \ldots, z_{n}^{\beta_{n}}\right)$ equals $R_{\beta}$, defined as in (2.6). Thus in view of Section 2.6, on $\widetilde{X}$ there is an $\widetilde{R}_{\beta}$ with support on $D_{1}$ such that $\pi_{*} \widetilde{R}_{\beta}=R_{\beta}$ and

$$
\widetilde{R}_{\beta}=\omega_{\beta} \wedge \bar{\partial} \frac{1}{\sigma_{1}^{n \operatorname{ord}_{D_{1}}\left(\mathfrak{m}^{\beta}\right)} \cdots \sigma_{N}^{n \operatorname{ord}_{D_{N}}\left(\mathfrak{m}^{\beta}\right)}},
$$

where $\omega_{\beta}$ is smooth.

Let $\widetilde{R}_{\alpha}=\pi^{*}\left(z^{\beta-\alpha}\right) \widetilde{R}_{\beta}$. Then $\widetilde{R}_{\alpha}$ has support on $D_{1}$ and by $(2.2), \pi_{*} \widetilde{R}_{\alpha}=R_{\alpha}$. Moreover

$$
\widetilde{R}_{\alpha} \wedge \pi^{*} d z=\omega \wedge \bar{\partial} \frac{1}{\sigma_{1}^{a_{1}} \cdots \sigma_{N}^{a_{N}}},
$$

where $a_{i}=n \operatorname{ord}_{D_{i}}\left(\mathfrak{m}^{\beta}\right)-\operatorname{ord}_{D_{i}}\left(z^{\beta-\alpha}\right)-\operatorname{ord}_{D_{i}}(d z)$ and $\omega$ is smooth. A direct computation gives that $\operatorname{ord}_{D_{i}}(d z) \geq \operatorname{ord}_{D_{i}}\left(z^{1}\right)-1$. Since $n \operatorname{ord}_{D_{1}}\left(\mathfrak{m}^{\beta}\right)=\operatorname{ord}_{D_{1}}\left(z^{\beta}\right)$, see Example 2.2, it follows that

$$
a_{1}=\operatorname{ord}_{D_{1}}\left(z^{\beta}\right)-\operatorname{ord}_{D_{1}}\left(z^{\beta-\alpha}\right)-\operatorname{ord}_{D_{1}}(d z) \leq \operatorname{ord}_{D_{1}}\left(z^{\alpha-1}\right)+1 \leq \operatorname{ord}_{D_{1}}(M),
$$

cf. (3.1).

That $D=\sum_{i=1}^{N} r_{i} D_{i}$ has simple normal crossings support means that at $x \in \tilde{X}$, we can choose coordinates $s_{1}, \ldots, s_{n}$ such that for some $p, \pi^{-1}(0)=\left\{s_{1} \cdots s_{p}=0\right\}$ and for each $i$ either $x \notin D_{i}$ or $D_{i}=\left\{s_{j}=0\right\}$ for some $j$. Thus, we may assume that at $x$, for $i=1, \ldots, p, \sigma_{i}=s_{i} \sigma_{i}^{\prime}$, where $\sigma_{i}^{\prime}$ does not vanish at $x$, and moreover $\sigma_{p+1}, \ldots, \sigma_{N}$ do not vanish at $x$. Since $a_{1} \leq r_{1}=\operatorname{ord}_{D_{1}}(M)$, in view of Example 2.1,

$$
\mathbf{1}_{D_{1}} \bar{\partial} \frac{1}{\sigma_{1}^{a_{1}} \cdots \sigma_{N}^{a_{N}}}=\mathbf{1}_{D_{1}} \sigma_{1}^{r_{1}-a_{1}} \cdots \sigma_{N}^{r_{N}-a_{N}} \bar{\partial} \frac{1}{\sigma_{1}^{r_{1}} \cdots \sigma_{N}^{r_{N}}} .
$$

Let $\eta_{\alpha}$ be the semi-meromorphic form $\eta_{\alpha}=\sigma_{1}^{r_{1}-a_{1}} \cdots \sigma_{N}^{r_{N}-a_{N}} \omega$. Since $\widetilde{R}_{\alpha}$ has support on $D_{1}$, it follows from (3.2) and (3.3) that

$$
\widetilde{R}_{\alpha} \wedge \pi^{*} d z=\eta_{\alpha} \wedge \bar{\partial} \frac{1}{\sigma_{1}^{r_{1}} \cdots \sigma_{N}^{r_{N}}} .
$$
form.

Now, let $\eta_{\tau}=c_{\tau} \eta_{\alpha}$ and $\eta=\sum_{\tau \in X_{n}} \eta_{\tau} e_{\tau} \otimes e_{\emptyset}^{*}$. Then $\widetilde{R}_{\tau}$ and $\widetilde{R}$ are of the desired

By using the description of residue currents of general, not necessarily Artinian, monomial ideals in [19, Section 5], it should be possible to extend Theorems 1.1 and 3.1 to this setting, although the formulations would become slightly more complicated. However, the arguments above rely heavily on the explicit descriptions of the log-resolution of a monomial ideal $M$ and the residue current $R$ of a cellular resolution of $M$, as well as the explicit description of Bochner-Martinelli residue currents, and it does not seem obvious how to extend them to non-monomial ideals.

In [16], Lazarsfeld and Lee proved that multiplier ideals are very special among integrally closed ideals by proving that the maps $\varphi_{j}$ in a free resolution do not vanish to high order in a certain sense. It might happen that in a similar way, $R$ has small singularities, in the sense that it is the pushforward of a current $\widetilde{R}$ that satisfies (1.6), only for a restricted class of integrally closed ideals.

We finally remark that if the residue current $R$, associated with a general ideal $\mathfrak{a} \subset \mathcal{O}_{0}^{n}$, is the pushforward of a current $\widetilde{R}$ of the form (1.4), then, in general, the exponents $a_{i}$ in (1.5) have to be (at least) like $n r_{i}$, where $r_{i}$ is as in the introduction. Indeed, assume that for some $v \in \mathbf{N}, a_{i} \leq v r_{i}$ for each $i$, and take $g \in \overline{\mathfrak{a}}^{v}$. Then $\pi^{*} g$ is divisible by $\sigma$ and thus $g R=0$, cf. the arguments after (1.5). It follows that $\overline{\mathfrak{a}}^{v} \subset \mathfrak{a}$. The classical Briançon-Skoda theorem [18] asserts that this inclusion holds for $v=\min (n, m)$, where $m$ is the minimum 
number of generators. This theorem is sharp and therefore in general the $a_{i}$ need to be at least like $n r_{i}$, cf. Example 2.3.

Acknowledgments I would like to thank Mats Andersson for valuable discussions on the topic of this paper. I am also very grateful to the referee for the careful reading, for pointing out some obscurities and mistakes in a previous version of this paper, and for several useful comments and suggestions.

Funding Information The author was partially supported by the Swedish Research Council.

Open Access This article is distributed under the terms of the Creative Commons Attribution 4.0 International License (http://creativecommons.org/licenses/by/4.0/), which permits unrestricted use, distribution, and reproduction in any medium, provided you give appropriate credit to the original author(s) and the source, provide a link to the Creative Commons license, and indicate if changes were made.

\section{References}

1. Andersson, M.: Residue currents and ideals of holomorphic functions. Bull. Sci. Math. 128(6), 481-512 (2004)

2. Andersson, M., Samuelsson, H.: A Dolbeault-Grothendieck lemma on complex spaces via Koppelman formulas. Invent. Math. 190(2), 261-297 (2012)

3. Andersson, M., Wulcan, E.: Residue currents with prescribed annihilator ideals. Ann. Sci. École Norm. Sup. 40(6), 985-1007 (2007)

4. Andersson, M., Wulcan, E.: Decomposition of residue currents. J. Reine Angew. Math. 638, 103-118 (2010)

5. Andersson, M., Wulcan, E.: Global effective versions of the Briançon-Skoda-Huneke theorem. Invent. Math. 200(2), 607-651 (2015)

6. Andersson, M., Wulcan, E.: Direct images of semi-meromorphic currents. Ann. Inst. Fourier (Grenoble) 68(2), 875-900 (2018)

7. Berenstein, C., Gay, R., Vidras, A., Yger, A.: Residue Currents and Bezout Identities. Progress in Mathematics, vol. 114. Birkhäuser, Basel (1993)

8. Bayer, D., Sturmfels, B.: Cellular resolutions of monomial modules. J. Reine Angew. Math. 502, 123140 (1998)

9. Coleff, N.R., Herrera, M.E.: Les Courants Résiduels Associés à une Forme Méromorphe. Lecture Notes in Mathematics, vol. 633. Springer, Berlin (1978)

10. Fulton, W.: Introduction to Toric Varieties. Annals of Mathematics Studies, vol. 131. Princeton University Press, Princeton (1993)

11. Herrera, M., Lieberman, D.: Residues and principal values on complex spaces. Math. Ann. 194, 259294 (1971)

12. Huneke, C., Swanson, I.: Integral Closure of Ideals, Rings, and Modules. London Mathematical Society Lecture Note Series, vol. 336. Cambridge University Press, Cambridge (2006)

13. Jonsson, M., Wulcan, E.: On Bochner-Martinelli residue currents and their annihilator ideals. Ann. Inst. Fourier (Grenoble) 59(6), 2119-2142 (2009)

14. Lärkäng, R., Wulcan, E.: Residue currents and fundamental cycles. Indiana Univ. Math. J. 67(3), 1085$1114(2018)$

15. Lazarsfeld, R.: Positivity in Algebraic Geometry. II. Ergebnisse der Mathematik und ihrer Grenzgebiete. 3. Folge. A Series of Modern Surveys in Mathematics [Results in Mathematics and Related Areas. 3rd Series. A Series of Modern Surveys in Mathematics], vol. 49. Springer, Berlin (2004)

16. Lazarsfeld, R., Lee, K.: Local syzygies of multiplier ideals. Invent. Math. 167(2), 409-418 (2007)

17. Passare, M., Tsikh, A., Yger, A.: Residue currents of the Bochner-Martinelli type. Publ. Mat. 44(1), 85117 (2000)

18. Skoda, H., Briançon, J.: Sur la clôture intégrale d'un idéal de germes de fonctions holomorphes en un point de $\mathbf{C}^{n}$. C. R. Acad. Sci. Paris Sér. A 278, 949-951 (1974)

19. Wulcan, E.: Residue currents constructed from resolutions of monomial ideals. Math. Z. 262(2), 235253 (2009)

Publisher's Note Springer Nature remains neutral with regard to jurisdictional claims in published maps and institutional affiliations. 